CASE REPORT

\author{
A.A. Lasboo \\ M.C. Hurley \\ M.T. Walker \\ D. Surdell \\ J.K. Song \\ J.M. Rosenow
}

A. Shaibani

\section{Emergent Image-Guided Treatment of a Large CSF Leak to Reverse "In-Extremis" Signs of Intracranial Hypotension}

\begin{abstract}
SUMmARY: We report the use of an emergent, targeted fibrin spinal epidural blood patch with subarachnoid saline infusion to rapidly reverse "in-extremis" clinical and imaging signs of posteriorfossa coning brought about by acute-on-chronic intracranial hypotension, itself consequent to a cervicothoracic CSF leak. Treatment resulted in a dramatic recovery and eventual discharge with return to normal lifestyle and occupation. The clinical and imaging danger signs are reviewed; fibrin patch technique and potential pitfalls in postprocedure management are analyzed.
\end{abstract}

$S$ pontaneous intracranial hypotension $(\mathrm{SIH})$ is characterized by low CSF pressure and postural headaches. ${ }^{1}$ Severe associated neurologic dysfunction including encephalopathy, though rare, has been reported. ${ }^{2-5}$ We describe a case of a patient with SIH with progressive neurologic deterioration and documented brain stem "coning" on head MR imaging, culminating in coma and incipient cardiopulmonary arrest. An emergent lumbar subarachnoid saline infusion and epidural fibrin patch, targeted to the region of the CSF leakage, resulted in an immediate stabilization with a full and persistent recovery.

\section{Case Report}

A 46-year-old man presented with severe orthostatic headaches, neck pain, nausea, and vomiting, worsening during 4 months. He recalled "straining" his back when bending over to lift a heavy object at his workplace 2 to 3 weeks before his symptom onset. MR of the head revealed diffuse thickening and enhancement of the pachymeninges suspicious for SIH, and he was transferred to our institution for work-up of a possible CSF leak.

On admission, results of his neurologic examination were normal. Opening pressure was low on lumbar puncture (LP), and repeat MR imaging confirmed diffuse pachymeningeal enhancement, as well as hemispheric subdural hematomas and mild descent of the cerebellar tonsils. Without symptomatic response to bed rest, intravenous fluid, and analgesics, the patient underwent a 10-mL lumbar epidural blood patch (EBP) to empirically treat the likely dural tear. This procedure relieved his symptoms, and he was discharged within 24 hours to be observed in the outpatient clinic.

He returned after 10 days with worsening headaches, which had recurred 2 days after his discharge. Brain CT scan showed enlargement of the subdural hematoma and increased effacement of the basal cisterns, particularly at the foramen magnum. Whole-spine MR imaging revealed extensive epidural fluid throughout the cervicothoracic region (Fig 1). Subsequent CT myelogram confirmed a CSF leak but localized poorly with contrast within the epidural space from $\mathrm{C} 1$

Received March 14, 2008; accepted after revision April 1

From the Departments of Radiology (A.A.L., M.C.H., M.T.W., D.S., A.S.) and Neurological Surgery (M.C.H., D.S., J.K.S., J.M.R., A.S.), The Feinberg School of Medicine and McGaw Medical Center, Northwestern University, Chicago, III.

Please address correspondence to Ali Shaibani, MD, Department of Radiology, 676 North St. Clair, Suite 800, Chicago, IL 60611; e-mail: AShaibani@nmff.org

DOI 10.3174/ajnr.A1157 to T8. We performed multilevel EBP with $30 \mathrm{~mL}$ of blood divided between the C3-C4, C7-T1, and T6-T7 levels. Again, he improved rapidly and was mobilized within 48 hours by the primary service.

Three days later, while still an inpatient, he sustained several acute episodes of unresponsiveness, followed by lethargy but without electroencephalographic evidence of seizure activity. Because of fluctuating mental status and enlarging subdural hematomas, continuous CSF leak was suspected, and he underwent a third high-volume (37$\mathrm{mL}$ ) EBP at the C7-T1, T6-T7, T11-T12, and L5-S1 levels. Resolution of lethargy was again followed by mobilization within 48 hours.

After 6 days, his vomiting and occipital headaches returned. A second CT myelography demonstrated epidural contrast from T1-T2 down to T10-T11, and his condition again progressed from lethargy to becoming unarousable in a few days. After intubation and right frontal intracranial pressure monitor insertion, he underwent LP and slow intrathecal infusion of $60-\mathrm{mL}$ saline at the level of L3-L4 to increase CSF pressure. A sudden elevation of his blood pressure and heart rate both resolved on immediate termination of the procedure. Concurrent intrathecal isotope injection did not further resolve the exact location of the leakage.

The next day, the patient underwent a percutaneous epidural patch (EP) with $13 \mathrm{~mL}$ of fibrin glue divided between C5 and C6, T1 and $\mathrm{T} 2$, and $\mathrm{T} 4$ and $\mathrm{T} 5$ levels and an autologous blood injection (35 $\mathrm{mL}$ ) at the same levels plus at the level of T9-T10 (Fig 2). In addition, burr-hole evacuation of the bilateral frontal and parietal subdural collections was performed. His neurologic condition significantly resolved after these interventions, and he was mobilized after 4 days and then discharged to home.

He returned 2 weeks later with recurrent headaches, nausea, vomiting, and enlargement of the subdural collections. Urgent craniotomy and re-evacuation of the larger right subdural collection failed to alleviate his symptoms, with a Glasgow Coma Scale of 4, nonreactive anisocoric pupils, decerebrate posturing to painful stimuli, and encephalopathic pattern on electroencephalography. Brain MR imaging demonstrated a tonsillar herniation $4.5 \mathrm{~mm}$ below the level of the foramen magnum, uncal herniation, effacement of the basal cisterns, right to left subfalcine midline shift ( $9 \mathrm{~mm}$ ), and kinking of the pontomesencephalic junction and basilar artery (Fig $3 A$ ).

Optimized CT myelography performed more rapidly after the contrast injection further localized the leak to be between T2 and T7, and he was transferred directly to the interventional suite. Emergent epidural patch with $12 \mathrm{~mL}$ of fibrin glue was performed at the C7-T1, $\mathrm{T} 2-\mathrm{T} 3, \mathrm{~T} 4-\mathrm{T} 5$, and T6 -7 levels along with a slow infusion of $12 \mathrm{~mL}$ of 

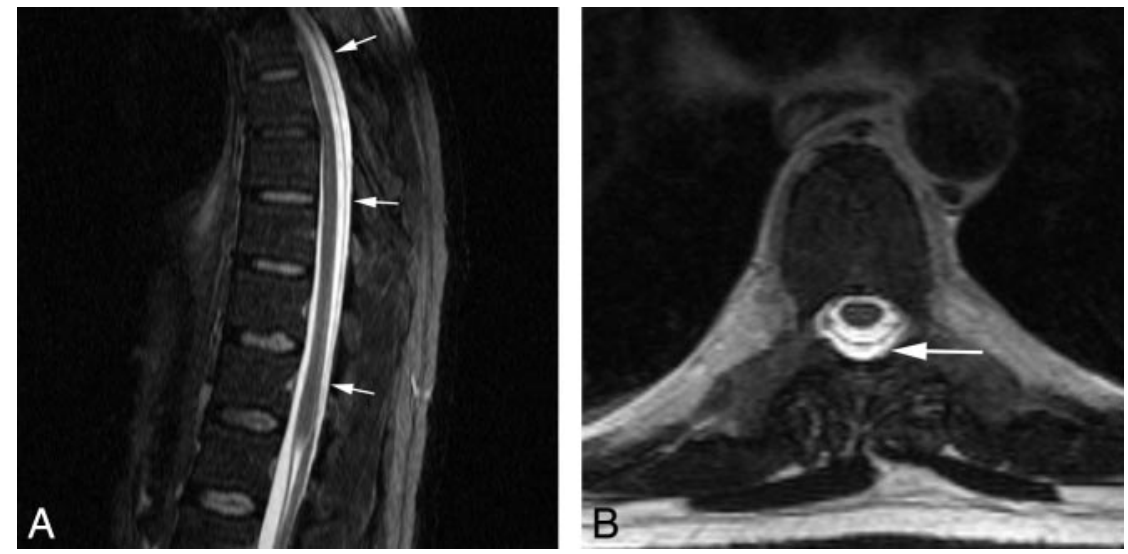

Fig 1. A, T2 sagittal MR imaging of the thoracolumbar spine with fat saturation shows a longitudinal posterior epidural fluid collection (arrows). B, T2 axial MR imaging through the T8 vertebra shows the posterior epidural fluid collection (arrow) extending around the sides of the thecal sac
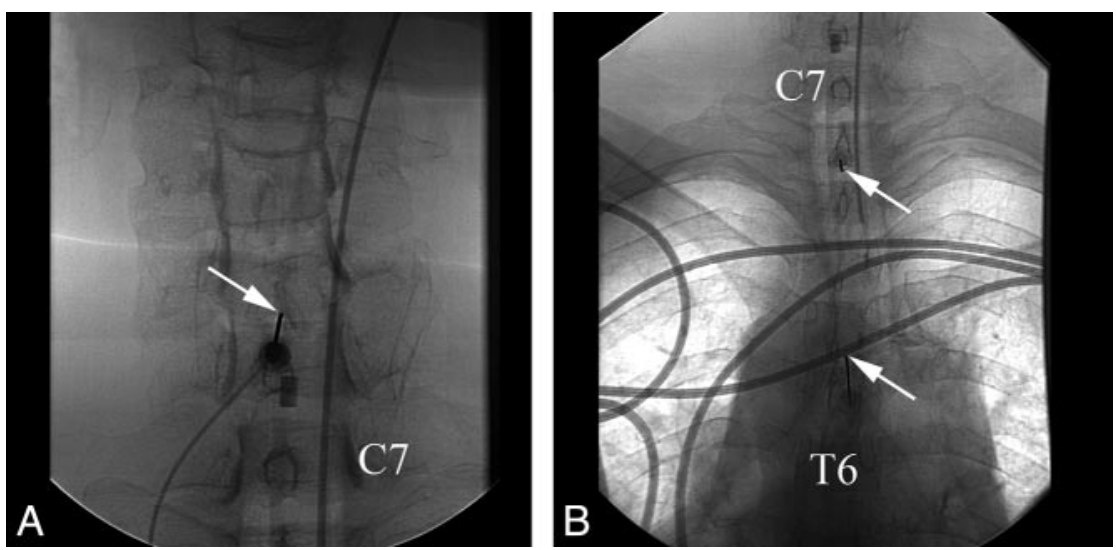

Fig 2. A, Spot anteroposterior (AP) radiograph of the lower cervical spine shows placement of the first C5-C6 sublaminar posterior epidural needle. B, Spot AP radiograph of the cervicodorsal spine shows placement of the second and third posterior epidural needles at the T1-T2 and T4-T5 levels.
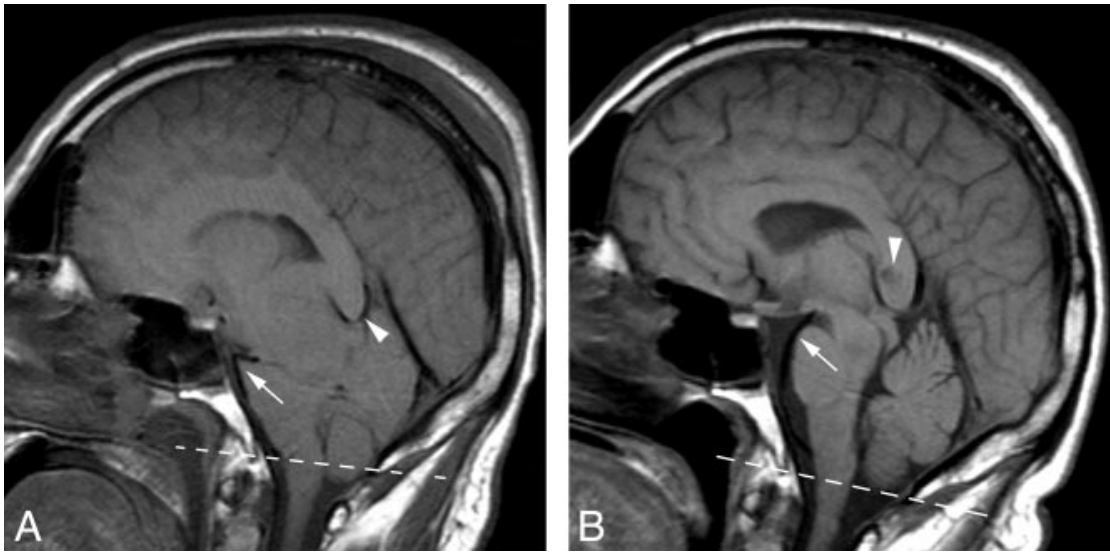

Fig 3. $A$, Midline noncontrast T1 sagittal brain MR imaging before emergent fibrin epidural patch demonstrates severe crowding of the posterior fossa with effacement of the basal cisterns and tonsillar descent through the foramen magnum (broken line). Note acute angulation of the distal basilar artery (arrow) and compression of the vein of Galen by the splenium of the corpus callosum (arrowhead. B, Repeat MR imaging with the same parameters performed 2 weeks after treatment and recovery. There is a normal appearance of the posterior fossa cisterns and gentle curvature of the basilar artery with resolution of distal kinking (arrow). Encephalomalacia in the splenium (arrowhead) from ischemia, which likely occurred because of compression of the splenial artery or draining veins.

saline into the subarachnoid space at the L3-L4 level while the intracranial pressure was monitored.

His neurologic status gradually improved after this procedure, with concomitant improvement in the subfalcine shift and resolution of the basal cistern effacement and tonsillar herniation (Fig 3B). Within 4 days, he was alert and oriented with normal results on neurologic examination and was gradually mobilized after 2 weeks of strict bed rest. On his most recent follow-up, 5 months after the discharge, he remained symptom-free with excellent recovery.

\section{Discussion}

In the case of our patient, near-terminal sequelae of severe SIH were reversed by emergent percutaneous subarachnoid infusion and a targeted fibrin epidural patch. A recurrent problem 
through this patient's course was the inability to pinpoint the exact site of the CSF leak in the presence of an extensive longitudinal epidural collection. We relied on strategic placement of multiple 20-gauge Tuohy epidural needles to cover the length of extravasated contrast documented on CT myelography and injection of a large volume of autologous blood ( $\geq 30$ $\mathrm{mL})$. We placed needles on alternate sides of the midline, assuring even bilateral distribution and avoiding potential compartmentalization by a midline plica. ${ }^{6}$

We usually attempt 2 autologous EBPs before trying fibrin glue, the use of which has been described previously. ${ }^{7}$ We often combine 6 to $12 \mathrm{~mL}$ of fibrin glue targeted to the likely leakage site, with an additional autologous blood patch for a total volume of 20 to $40 \mathrm{~mL}$. We recommend 3 to 10 days of bed rest for patients with large leaks. In our patient, our initial EBPs were only briefly successful, likely because of a combination of a large tear and early mobilization.

Careful intrathecal saline infusion and pressure normalization serve 2 purposes. First, low intrathecal pressure in a decompressed subarachnoid space may reduce diagnostic contrast extravasation during myelography. Second, normalization of intraspinal and intracranial pressure hastens the relief of symptomatic SIH, critical in this case given the grave and deteriorating condition of the patient.

Subdural hematomas occurring secondary to SIH usually resolve spontaneously after the CSF leak is repaired. ${ }^{8}$ In our patient, a sizeable hematoma was evacuated given the degree of tonsillar herniation and the desperate clinical situation. He continued to deteriorate after the evacuation.

\section{Conclusion}

Emergent percutaneous subarachnoid saline infusion and an epidural fibrin patch targeted to the site of an epidural CSF leak can reverse the advanced stages of brain stem herniation and lead to a full and functional recovery. Patients who are treated should complete a full course of strict supine bed rest.

\section{References}

1. Mokri B, Piepgras DG, Miller GM. Syndrome of orthostatic headaches and diffuse pachymeningeal gadolinium enhancement. Mayo Clin Proc 1997;72:400-13

2. Beck CE, Rizk NW, Kiger LT, et al. Intracranial hypotension presenting with severe encephalopathy. Case report. J Neurosurg 1998;89:470-73

3. Evans RW, Mokri B. Spontaneous intracranial hypotension resulting in coma. Headache 2002;42:159-60

4. Whiteley W, Al-Shahi R, Myles L, et al. Spontaneous intracranial hypotension causing confusion and coma: a headache for the neurologist and the neurosurgeon. Br J Neurosurg 2003;17:456-58

5. Pleasure SJ, Abosch A, Friedman J, et al. Spontaneous intracranial hypotension resulting in stupor caused by diencephalic compression. Neurology 1998;50:1854-57

6. Mathis JM, Shaibani A, Wakhloo AK. Spine anatomy. In: Mathis JM, ed. ImageGuided Spine Interventions. New York: Springer-Verlag; 2004:14-15

7. Schievink WI, Moser FG, Pikul BK. Reversal of coma with an injection of glue. Lancet 2007;369:1402

8. Schievink WI, Maya MM, Moser FG, et al. Spectrum of subdural fluid collections in spontaneous intracranial hypotension. J Neurosurg 2005;103:608-13 


\section{Erratum}

In the article "Emergent Image-Guided Treatment of a Large CSF Leak to Reverse 'In-Extremis' Signs of Intracranial Hypotension" (AJNR Am J Neuroradiol 2008;29:1627-29), the last name of the first author was incorrectly provided as "Lasboo." It should be corrected to "Aghaei Lasboo."

DOI 10.3174/ajnr.A2059 